\title{
Strain and Temperature Discrimination Using Modal Interferometry in Bragg Fibers
}

\author{
Orlando Frazão, Luís M. N. Amaral, José M. Baptista, Philippe Roy, Raphael Jamier, and Sébastien Février
}

\begin{abstract}
The strain and temperature sensing characteristics of a modal interferometer based on two Bragg fibers have been investigated. The special nature of this sensor is that the two Bragg fibers used present a different external cladding shape. It appears that the sensitivity to the sensing parameters are different for the two Bragg fibers, which makes it possible to fabricate several sensing configurations based on the combination of these two Bragg fibers for strain and temperature discrimination.
\end{abstract}

Index Terms-Bragg fiber, interferometer, modal interferometry, strain and temperature measurement.

\section{INTRODUCTION}

B RAGG fibers, whose theoretical model was proposed in 1978 by Yeh et al. [1], are photonic bandgap fibers composed of a low index core surrounded by concentric dielectric layers of high and low index. Many studies have been conducted on these bandgap fibers due to their unusual properties (low bending loss, dispersion properties, spectral filtering, etc.). For instance, such a fiber can propagate a single large Gaussian mode and, therefore, be useful for power delivery [2], [3] or high-power lasers [4]. Recently, the first polarizationmaintaining photonic bandgap Bragg fiber has been demonstrated [5]. Furthermore, the chromatic dispersion curve can be tailored. Thus, these fibres can also be used in various applications such as supercontinuum generation [6] or dispersion compensation [7]. Regarding optical fiber sensing, such a bandgap fiber can also be an alternative solution for specific situations, as was demonstrated in a band rejection filter sensor configuration [8] or a surface-plasmon resonance sensor for refractive index measurement [9] or even as a temperature strain sensor [10].

In this letter, one proposes the utilization of two different pieces of Bragg fibers to perform modal sensing interferometry applied to the measurement of strain and temperature. Due to the fibers characteristics, the linearly polarized fundamental mode LP01 and the high order mode LP02 can be excited simultaneously creating a modal interferometer in the 1550-nm region. The two different pieces of Bragg fibers were spliced in series and the return interferometric signals were discriminated using

Manuscript received June 24, 2010; revised July 27, 2010; accepted September 03, 2010. Date of publication September 13, 2010; date of current version October 15, 2010. This work was performed in the framework of European Program COST, Action 299.

O. Frazão, L. M. N. Amaral, and J. M. Baptista are with INESC Porto-Instituto de Engenharia de Sistemas e Computadores do Porto, 4169-007 Porto, Portugal, and also with Departamento de Física da Faculdade de Ciências da Universidade do Porto, 4169-007 Porto, Portugal (e-mail: ofrazao@inescporto.pt).

P. Roy, R. Jamier, and S. Février are with Xlim, Photonics Department, UMR 6172 CNRS University of Limoges, 87060 Limoges, France.

Color versions of one or more of the figures in this letter are available online at http://ieeexplore.ieee.org.

Digital Object Identifier 10.1109/LPT.2010.2076357

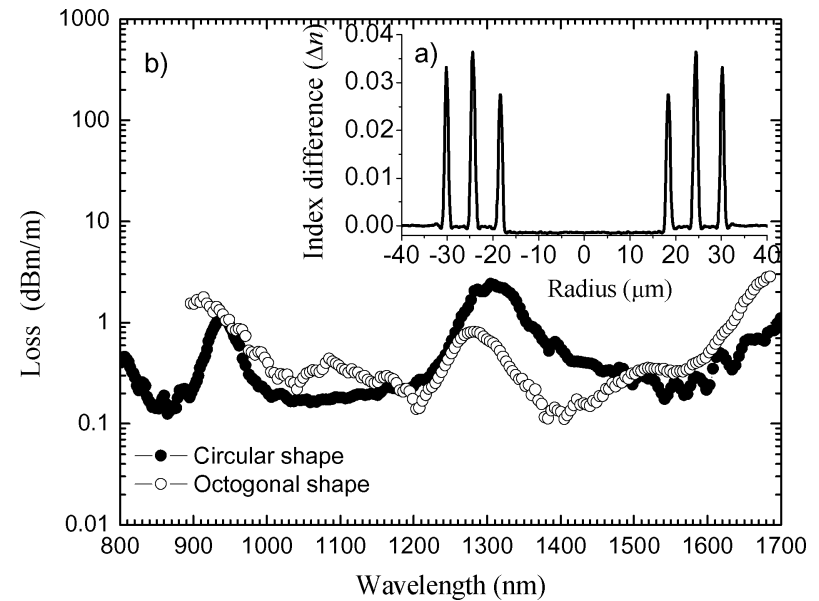

Fig. 1. Measured refractive index profile (a) and measured transmission spectra (b) of the two Bragg fibers.

coherence addressing and the interrogation was performed applying a heterodyne technique.

\section{EXPERIMENTAL RESULTS}

Bragg fibers, which are anti-resonant reflecting optical waveguides (ARROW), allow the propagation of light in a core with a refractive index smaller than the microstructured periodic cladding one. Light, launched in the fiber core, is coherently reflected onto the cladding and then is trapped in the fiber core for certain wave vectors. Strictly, we do not have modal propagation, but if the number of layers is not too small the propagation approaches a modal-type one. In these experiments, both fibers used have been drawn from the same preform to an external diameter of $125 \mu \mathrm{m}$. The difference between the two fibers is the pure silica external cladding shape. A first fiber drawing was made to obtain the first fiber. Then the external shape of the preform has been mechanically modified so as to have an octagonal shape. A second fiber drawing was then carried out leading to the second fiber. The cross-section images of the both fibers are shown in the inset of Fig. 2. The both fibers are characterized by a depressed-index core surrounded by three bilayers of high and low index. The index difference between the core and the pure silica low-index layers is close to $1 \times 10^{-3}$ [see Fig. 1(a)]. The core diameters of the fibers are similar, $\sim 35.7 \mu \mathrm{m}$. Fig. 1(b) superimposes the attenuation spectra of the both fibers measured by the cut-back technique. Three low-loss bandgap regions can be observed. In the vicinity of $\lambda=1550 \mathrm{~nm}$ corresponding to the center of the first bandgap, the loss is $\sim 0.2 \mathrm{~dB} / \mathrm{m}$. The experimental setup of the sensing interferometer is shown in Fig. 2. The lead fibers are SMF 28. The sensing head consists of two pieces 


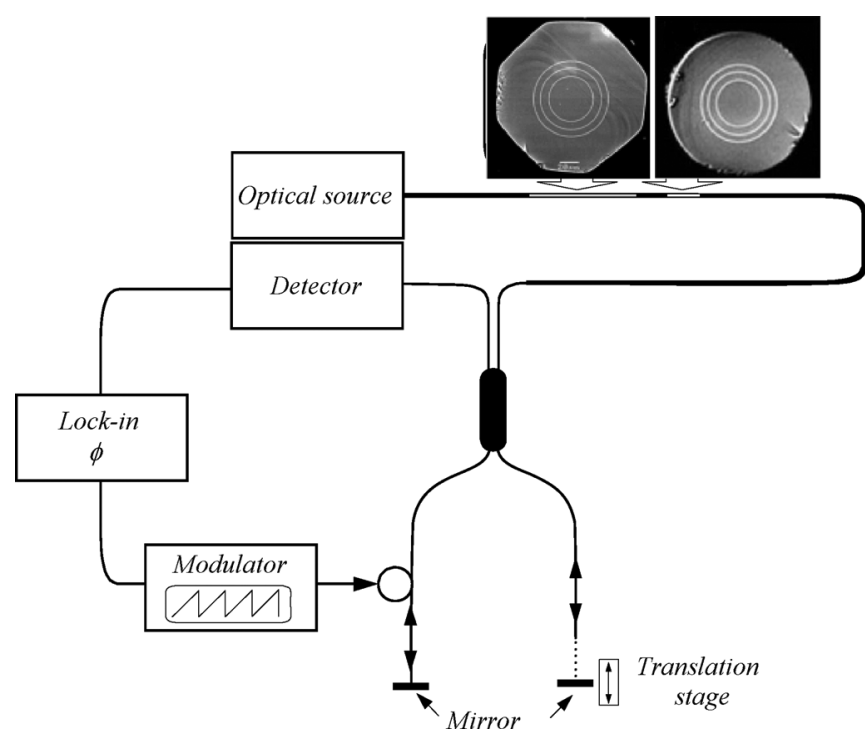

Fig. 2. Experimental setup showing the sensing head and the receiving interferometer. Inset: cross-section of the both Bragg fibers.

of the both Bragg fibers with different lengths $(30 \mathrm{~mm}$ for the fiber with the circular external cladding shape and $350 \mathrm{~mm}$ for the octagonal one), with a small length of connecting standard single-mode fiber (SMF 28) put in between them. The loss splice is approximately $1.5 \mathrm{~dB}$ for two splices between the SMF 28. The total sensing head loss is $3 \mathrm{~dB}$. By nature, a photonic bandgap fiber is a multimode waveguide. The single-mode behavior is reached by making a modal discrimination based on the confinement loss of each mode. Thus, a Bragg fiber exhibits single-mode propagation only if the losses of the high order modes are higher than those of the fundamental mode. Due to the short lengths of Bragg fibers used in these experiments, a multimode propagation is expected. Calculations using the full-vector transfer matrix method have confirmed that the both pieces of Bragg fibers propagate the two quasi-modes noted LP01 and LP02 [9]. Therefore, each piece of Bragg fiber constitutes a modal interferometer based on these two quasi-modes, which means that the sensing head has two-in series modal interferometers with different path imbalances due to the different fiber lengths. These path imbalances are much larger than the EDFA source coherence length, meaning that in the time domain at the end of the return SMF 28 there is no noticeable interference. This situation changes if a second interferometer is introduced in the reception region to implement the white-light readout technique. As shown in Fig. 2, the light returned from the sensing head is injected into a receiver Michelson interferometer with an open air path in one of the arms, which is used to match the optical path difference of one of the modal interferometers in the sensing head. In this way, coherence addressing of that interferometer is performed and an interferometric signal is generated at the input of the photodetector. Applying a saw tooth waveform with proper amplitude to a piezoelectric transducer (PZT) fiber stretcher in one arm of the receiving interferometer, an electric carrier can be obtained through electrical filtering of the detected signal, whose phase is a replica of the phase of the tandem modal interferometer. This phase is then measured using a lock-in amplifier. To read the signal from the second modal

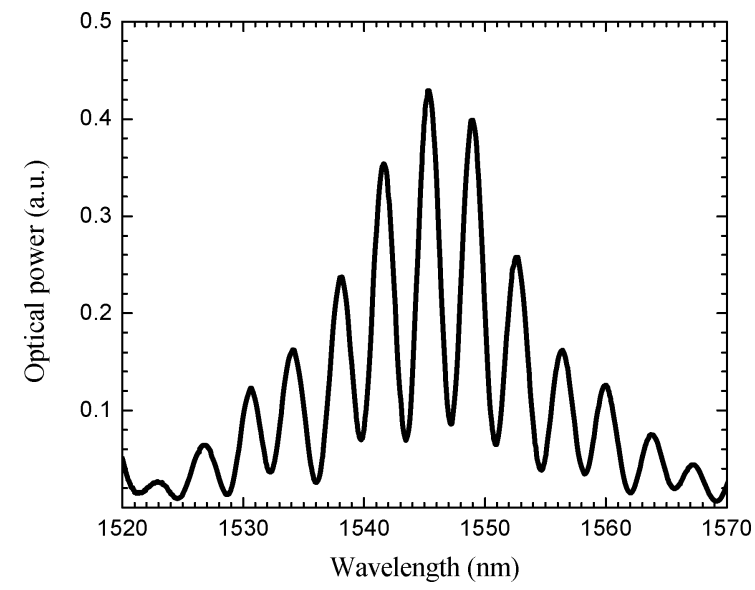

Fig. 3. Channeled spectrum of the interferometric sensing head.
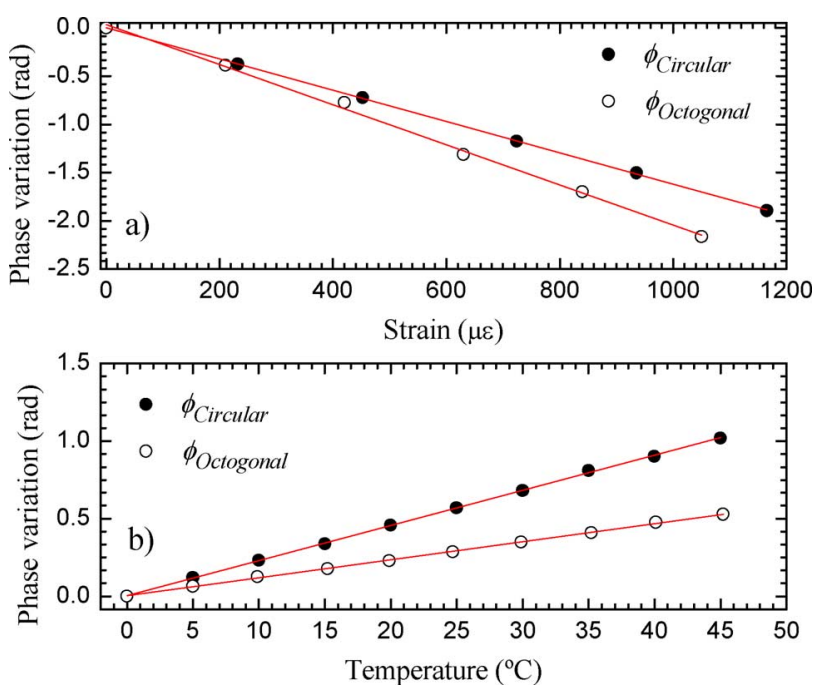

Fig. 4. Phase variation of the interferometric sensing system when strain (a) and temperature (b) are applied to the sensing head.

interferometer, the Michelson interferometer is tuned to it by changing its path imbalance. To check the interferometric signal arising from a Bragg fiber modal interferometer, the channeled spectrum at the input of the processing interferometer was obtained when only the piece of the Bragg fiber with the octagonal external cladding shape was present in the sensing head. Fig. 3 shows this measured spectrum, which is typical of a two beam interferometer, indicating that the structure proposed works as a modal interferometer involving essentially the two quasimodes LP01 and LP02 of the Bragg fiber. After, the sensing head integrating the two pieces of Bragg fibers was characterized for strain and temperature. In both cases, the phase data from each modal interferometer was obtained tuning the path imbalance of the processing interferometer to that of the modal interferometer. The results obtained are plotted in Fig. 4. It is important to underline that the readout phase signal is the phase difference between the light that propagates in the two modes excited by the input standard single-mode fiber. Therefore, the accumulated phase difference shall essentially be due to different modal elasto-optic coefficients (for the case of strain) and different modal thermo-optic coefficients (for the case of temperature). In the case of strain, the data shown 


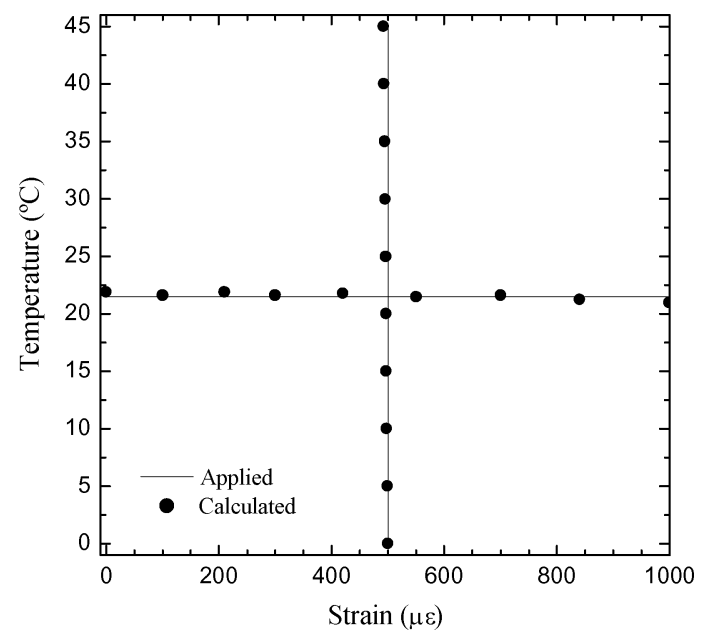

Fig. 5. Sensor output as determined by (2) for applied temperature and strain.

in Fig. 4(a) indicate sensitivity magnitudes of $1.6 \mathrm{mrad} / \mu \varepsilon$ (for the Bragg fiber with the circular external cladding shape) and $2.1 \mathrm{mrad} / \mu \varepsilon$ (for the Bragg fiber with the octagonal one). For temperature, the coefficients are $22 \mathrm{mrad} /{ }^{\circ} \mathrm{C}$ (for the circular Bragg fiber) and $11 \mathrm{mrad} /{ }^{\circ} \mathrm{C}$ (for the octagonal one). The strain coefficients are first-order independent of the fiber length, but the temperature coefficients scale approximately with the length of the modal interferometers. Therefore, by normalizing, the temperature coefficients are $7.3 \mathrm{mrad} / \mathrm{cm}^{\circ} \mathrm{C}$ (for the circular Bragg fiber) and $0.3 \mathrm{mrad} / \mathrm{cm}^{\circ} \mathrm{C}$ (for the octagonal one). The difference in the strain and temperature coefficients of the two Bragg fibers, more particularly for the case of temperature where the difference is by a factor of 24 , are certainly due to different frozen internal stresses applied to the fibers during their fabrication process. This is a topic where further investigation is on target. This difference in the sensitivity coefficients opens the possibility to perform with this sensing head simultaneous measurement of strain and temperature. Indeed, with the coefficients derived from Fig. 4 it can be written

$$
\left[\begin{array}{c}
\Delta T \\
\Delta \varepsilon
\end{array}\right]=\frac{1}{28.6}\left[\begin{array}{cc}
-2.1 & 1.6 \\
11 & -22
\end{array}\right]\left[\begin{array}{c}
\Delta \phi_{\text {Circular }} \\
\Delta \phi_{\text {Octogonal }}
\end{array}\right]
$$

This relation permits from the measured phase variation of the two modal interferometers to derive the associated variations of temperature and strain. The performance of this simultaneous measurement configuration was experimentally determined by undertaking strain variations in a range of $1000 \mu \varepsilon$; at a fixed temperature $\left(22^{\circ} \mathrm{C}\right)$ and the other way around, i.e., temperature variations in a range of $50{ }^{\circ} \mathrm{C}$ for a specific applied strain $(500 \mu \varepsilon)$, as shown in Fig. 5. From these results, root-mean-square deviations of $\pm 0.9^{\circ} \mathrm{C}$ and $\pm 8 \mu \varepsilon$ were determined for temperature and strain measurements, respectively. In terms of performance comparison, this configuration presents a matrix condition number of 21.3, which is a better result when comparing with the configurations presented in [11].

\section{CONCLUSION}

It was demonstrated that it is possible to build up modal interferometry with Bragg fibers having a differential phase sensitive to strain and temperature variations. Two pieces of Bragg fibers were studied, one with a circular external cladding shape and the other with an octagonal one. They showed distinct differential phase sensitivity to those measured. A sensing head with two modal interferometers in series was implemented, addressed in coherence and with heterodyne interrogation. The results obtained indicate the feasibility to use this sensing structure to perform the functionality of simultaneous measurement of strain and temperature. Two important features of this work are the use of Bragg fibers as interferometers and the different achieved sensitivities with different Bragg fiber cladding geometries.

\section{ACKNOWLEDGMENT}

The authors acknowledge S. L. Semjonov, M. E. Likhachev, M. M. Bubnov, and E. M. Dianov from the Fiber Optics Research Center, Moscow, and V. F. Khopin, M. Y. Salganskii, and A. N. Guryanov from the Institute of Chemistry of High Purity Substances, and N. Novgorod for the fabrication of the fiber samples.

\section{REFERENCES}

[1] P. Yeh, A. Yariv, and E. Marom, "Theory of Bragg fiber," J. Opt. Soc. Amer., vol. 68, no. 9, pp. 1196-1201, Sep. 1978.

[2] S. Février, P. Viale, F. Gerome, P. Leproux, P. Roy, J.-M. Blondy, B. Dussardier, and G. Monnom, "Very large effective area singlemode photonic bandgap fiber," Electron. Lett., vol. 39, no. 2, pp. 1240-1242, Aug. 2003.

[3] S. Février, R. Jamier, J.-M. Blondy, S. L. Semjonov, M. E. Likhachev, M. M. Bubnov, E. M. Dianov, V. F. Khopin, M. Y. Salganskii, and A. N. Guryanov, "Low-loss singlemode large mode area all-silica photonic bandgap fiber," Opt. Express, vol. 14, no. 2, pp. 562-569, Jan. 2006.

[4] S. Février, D. D. Gaponov, P. Roy, M. E. Likhachev, S. L. Semjonov, M. M. Bubnov, E. M. Dianov, M. Y. Yashkov, V. F. Khopin, M. Y. Salganskii, and A. N. Guryanov, "High-power photonic-bandgap fiber laser," Opt. Lett., vol. 33, no. 9, pp. 989-991, May 2008.

[5] M. E. Likhachev, A. D. Pryamikov, D. A. Gaponov, M. M. Bubnov, M. Y. Salganskii, V. F. Khopin, A. N. Guryanov, and S. Février, "Polarization-maintaining photonic bandgap Bragg fiber," Opt. Lett., vol. 34, no. 9, pp. 1366-1368, May 2009.

[6] R. Jamier, S. Février, N. Ducros, M. E. Likhachev, and M. Y. Salganskii, "Tight control of the spectral broadening obtained by nonlinear conversion in a photonic bandgap fiber," in Proc. CLEO/IQEC, Baltimore, MD, May 31- Jun. 5, 2009, Paper JWA53.

[7] F. Gérôme, S. Février, A. D. Pryamikov, J.-L. Auguste, R. Jamier, J.-M. Blondy, M. E. Likhachev, M. M. Bubnov, S. L. Semjonov, and E. M. Dianov, "Highly dispersive large mode area photonic bandgap fiber," Opt. Lett., vol. 32, pp. 1208-1210, May 2007.

[8] D. Chen, T. J. Yang, J. J. Wu, L. Shen, K.-L. Liao, and S. He, "Band-rejection fiber filter and fiber sensor based on a Bragg fiber of transversal resonant structure," Opt. Express, vol. 16, no. 21, pp. 16489-16495, Oct. 2008.

[9] L. Ma, T. Katagiri, and Y. Matsuura, "Surface-plasmon resonance sensor using silica-core Bragg fiber," Opt. Lett., vol. 34, no. 7, pp. 1069-1071, Apr. 2009.

[10] O. Frazão, J. M. Baptista, J. L. Santos, P. Roy, R. Jamier, and S. Février, "Bragg fibre for sensing applications," Proc. SPIE, vol. 7714, pp. 7714-7732, May 1-5, 2010.

[11] K. Kalli, A. G. Simpson, K. Zhou, L. Zhang, and I. Bennion, "Tailoring the temperature and strain coefficients of Type I and Type IA dual grating sensors-The impact of hydrogenation conditions," Meas. Sci. Technol., vol. 17, no. 5, pp. 949-954, May 2006. 